\title{
Myocardial Infarction Due to Essential Thrombocythemia
}

\author{
Bairong Chen, Mao Liu, Liyun Luo*, Yin Huang, Ani Wang, Xiufang Lin, Songbiao Li \\ Department of Cardiology, Fifth Affiliated Hospital of Sun Yat-Sen University, China \\ *Corresponding Author: zdwyresearch@163.com
}

Copyright (C) 2015 by authors, all rights reserved. Authors agree that this article remains permanently open access under the terms of the Creative Commons Attribution License 4.0 International License

\begin{abstract}
Essential thrombocythemia (ET) is a proliferative disorder of mature myeloid which may lead to persistently elevated platelet count. The clinical manifestations of ET include hemorrhagic and thrombotic complications, especially thrombus in the cerebral and peripheral arteries. However, coronary artery thrombosis in ET patient is rare. In this article, we report a 78-year-old woman who presented with acute myocardial infarction (AMI) as the first clinical sign of ET. Diagnostic coronary angiography revealed acute occlusion of the right coronary artery and percutaneous aspiration thrombectomy was performed successfully. The patient then received hydroxycarbamide and prednisone under supervision of a haematologist. She recovered very well during a follow-up more than three months. With this case, we hope that clinicians would always keep in mind that ET is an unusual cause of AMI and the patients with ET should be informed about the risk of fatal cardiac events.
\end{abstract}

Keywords Essential Thrombocythemia, Coronary Angiography, Acute Myocardial Infarction

\section{Introduction}

Essential thrombocythemia (ET) is a proliferative disorder of mature myeloid which may lead to persistently elevated platelet count [1]. The incidence rate for ET is about 2.5 to 3.0 cases per $10^{5}$ populations per year. The median age at diagnosis is 65 to 70 years, but the disease may occur at any age, especially in females. A V617F mutation in the gene encoding Janus kinase 2 (JAK2) in hematopoietic progenitor cells is usually found positive in ET patients. The clinical manifestations of ET include hemorrhagic and thrombotic complications, especially thrombotic complications in the cerebral, coronary, and peripheral vessels [2-3]. Both thrombosis and hemorrhage result from qualitative and quantitative defects of the platelets. However, coronary artery thrombosis in ET patient is rare. Moreover, acute myocardial infarction (AMI) caused by ET is often misdiagnosed because of its atypical symptoms and low incidence [4]. In this article, we shared a case who presented with AMI as the first clinical sign of ET.

\section{Brief Summary}

A 78-year-old woman with hypertension was admitted to emergency department because of severe and persistent chest pain for 3 hours. The electrocardiogram indicated sinus rhythm with 2-4mm ST-segment elevation in lead II, III, aVF, $V_{3 R}$ and $V_{4 R}$ (Fig. 1a). Serum troponin I peaked at 32 $\mathrm{ng} / \mathrm{ml}$. Diagnostic coronary angiography (CAG) revealed acute occlusion of the right coronary artery (Fig. 1b). Immediately, percutaneous aspiration thrombectomy was performed (Fig. 1c). Extensive intracoronary thrombus was aspirated out (Fig. 1d). Then, a TIMI grade 3 Flow was obtained. Therefore, no stents were implanted in the criminal coronary artery in this patient.

After the emergency $\mathrm{CAG}$, the blood routine test indicated that the platelet count was up to $1083.0 \times 109 / \mathrm{L}$. The bone marrow puncture results indicated thrombocytosis. Further, the JAK2 V617F gene mutation test was found positive in the gene studies. Finally, a diagnosis of AMI and ET was made. Accordingly, the patient received hydroxycarbamide and prednisone under supervision of a haematologist. She recovered well and her blood routine test results became normal several weeks later. During the follow-up more than three months, she remained free of symptoms and the blood routine tests were all normal. 

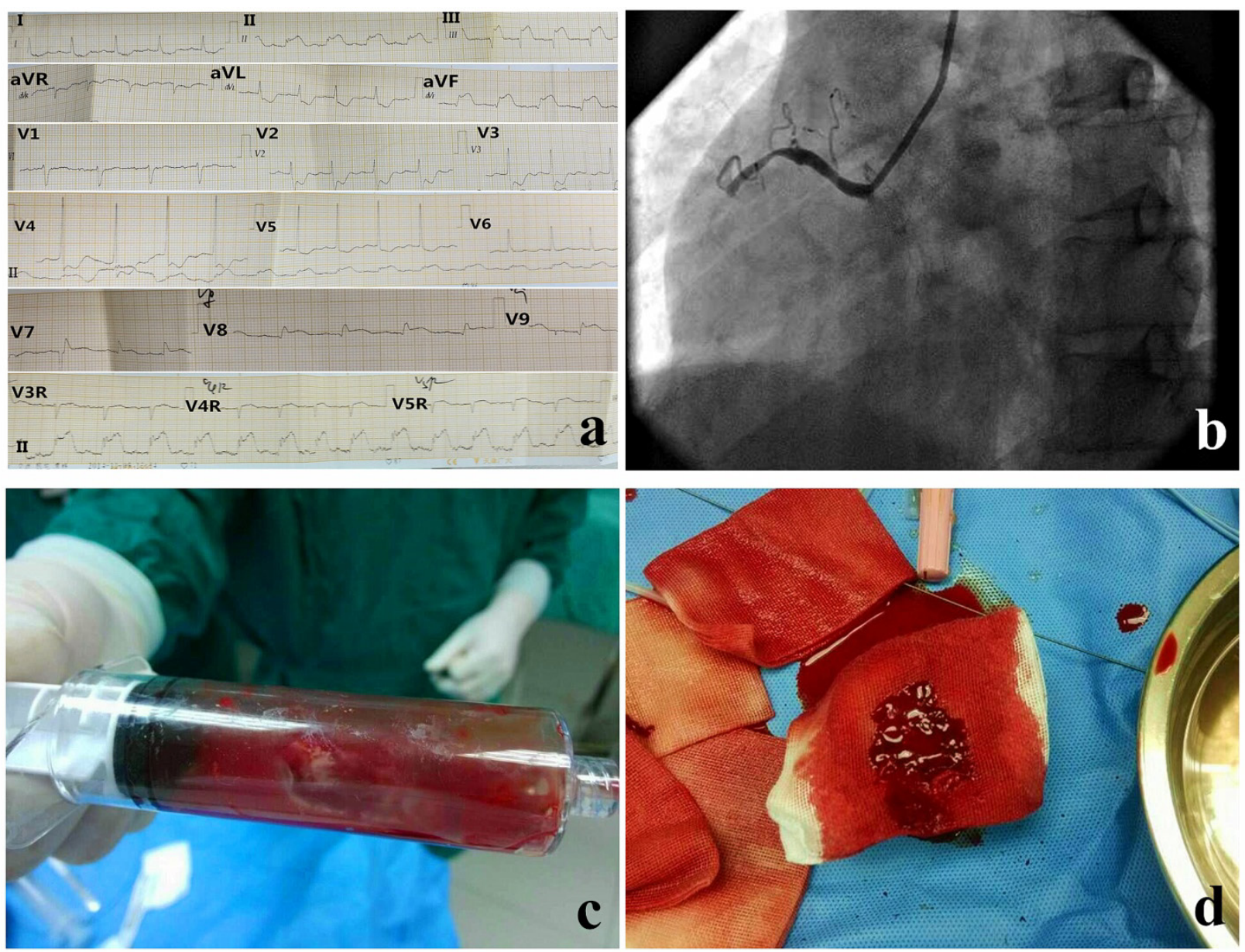

Figure 1. (a) The electrocardiogram showing sinus rhythm with 2-4mm ST-segment elevation in lead II, III, aVF, V3R and V4R. (b) CAG revealing acute occlusion of the right coronary artery. (c) Percutaneous aspiration thrombectomy was performed. (d) Extensive intracoronary thrombus was aspirated out.

\section{Conclusions}

We report a 78-year-old woman who presented with AMI as the first clinical sign of ET. Percutaneous aspiration thrombectomy was performed. She remained free of symptoms and the blood routine tests were all normal after the treatment of hydroxycarbamide and prednisone. With this case, we hope that clinicians would always keep in mind that ET is an unusual cause of AMI and the patients with ET should be informed about the risk of fatal cardiac events.

\section{Acknowledgements}

Equal contributions were made by Dr. B Chen \& Dr. M Liu to this work. The authors declare that they have no conflicts of interest concerning this article.

\section{REFERENCES}

[1] Singla A, Jagasia D, Garg M, et al. Acute ST-segment elevation myocardial infarction: A rare initial presentation of previously undiagnosed essential thrombocythemia. Platelets,2012,23:463-466.

[2] Brière JB.Essential thrombocythemia.Orphanet J Rare Dis. 2007 Jan 8;2:3

[3] Bhat T, Ahmed M, Baydoun H, et al. Acute ST-segment elevation myocardial infarction in a young patient with essential thrombocythemia: a case with long-term follow-up report. J Blood Med,2014,5:123-127.

[4] Gao W, Shen W, Luo X, et al. ST-segment elevation myocardial infarction in patient with essential thrombocythemia without associated risk. International Journal of Cardiology,2015,180:223-225. 